\title{
ON THF CORRECTION OF THE TRANSFERENCE NUMBERS OF THE IONS OF AN ELECTROLYTE.
}

\author{
Mr. W. R. Bousfield also contributed a paper "On the Correction \\ of the Transference Numbers of the Ions of an Electrolyte."
}

The recognition of the fact that the ions of an electrolyte carry with them certain amounts of water, which in some cases largely increase their bulk, led to the supposition that the transference numbers as determined by Hittorf and others were of little value. Thus Washburn, who endeavoured to determine the hydration of the ions by transference experiments in the presence of a non-electrolyte, says *:--

"In the usual method of calculating transference numbers the assumption is made that the solvent is absolutely stationary, that is, that no transfer of solvent takes place from one electrode to the other. If such a transfer of solvent takes place, the calculation of the transference number by the ordinary method is not justified and will not give the true transference number. In fact, the number so obtained does not represent any physical quantity. Only in dilute solutions, where the amount of solvent transferred becomes negligible in comparison with the total solvent present, will the ordinary transference number approach the true one."

Now, in a normal solution of lithium chloride about one-fifth of the whole water is in combination with the solute. One equivalent of $\mathrm{Li}$ ions transports with it about I 50 c.c. of water, and one equivalent of $\mathrm{Cl}$ ions about 5 o c.c. of water. Prima facie one would certainly think such large and unequal figures for water transport in a normal solition of $\mathrm{LiCl}$ would upset the transference numbers.

Washburn gives the results of his experiments with a $\mathrm{I} \cdot 3$ weight normal solution of $\mathrm{LiCl}$, which lead him to fix the ordinary transference number for this concentration at the figure 0.722 instead of $0.74 \mathrm{C}$, as given by Kohlrausch, and the true transference number at 0.694 .

It is difficult to criticize these experiments, because his experimental figures cannot easily be distinguished from the figures which are the results of deduction. It looks as if the method of calculation omitted consideration of the equation of continuity, which is, of course, a necessary factor. The matter being of so much importance, it seemed worth while to investigate the relation of the Hittorf number to the true transference number. The statement that the Hittorf number does not represent any physical quantity is somewhat misleading. It represents the ratio of the loss of cation in the cathode compartment to the weight of cation deposited at the cathode, which is an experimental figure of great impor-

* Technological Quarterly, 21, 288, I908. 
tance, from which the true transference number can be ascertained if the ionic volumes can be estimated with fair approximation. In the sequel it appears that the required correction is so small down to normal dilution as to be less than the experimental errors involved in transference experiments.

In former papers * I have shown how the approximate volumes of the ions may be calculated from the conductivity data. In the first three of those papers the method led to the deduction of the total volume in c.c of an equivalent of ions, including in the volume of the ion both the ionic nucleus and the combined water. In the last paper the result for $\mathrm{LiCl}$ was given in the form

$$
n=38 \boldsymbol{a}-\mathbf{I}_{4},
$$

where $n$ is the number of mols of water in combination with one equivalent of solute and $\boldsymbol{a}$ is the ionisation. Fither of these results can be used to estimate the approximate volume of an equivalent of ions of either kind with sufficient accuracy for the purposes of the correction which we are seeking, if the experimental Hittorf number can be relied upon. But it will be noticed that the comparative figures of Washburn and Kohlrausch above given involve a wide margin of experimental error.

At the risk of being too elementary it is desirable to state the theoretical steps involved, without leaving gaps in the reasoning which are puzzling to the student, though the professor will take them in his stride. We shall suppose the electrolyte to be contained in a rectangular trough of considerable length and of $\mathrm{x} \mathrm{sq.} \mathrm{cm}$. cross section. The electrodes are at the ends of the trough and may be supposed to be in the form of plates, I sq. $\mathrm{cm}$. in area. The current may be taken to be that due to a potential gradient of $\mathrm{I}$ volt per $\mathrm{cm}$. The resulting current is by definition the specific conductivity $k$ of the electrolyte, and is, by Ohm's law, equal to the reciprocal of the resistance of a $I-c m$. cube of the electrolyte.

Speaking in terms of a binary monovalent electrolyte, such as $\mathrm{LiCl}$. there occur two processions of the ions in opposite directions with velocities $U$ and V. In Fig. I the open circles represent positively charged

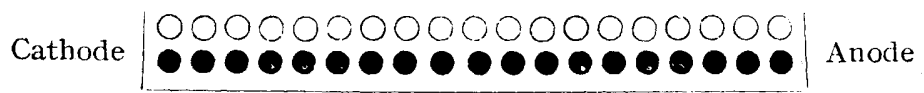

FIG. I.

cations and the black circles negatively charged anions existing in pairs. Fig. 2 represents diagrammatically the condition of things after

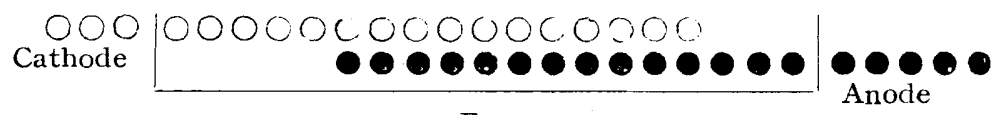

FIG. 2.

a time $t$ in which the cations have moved three places to the left, whilst the anions have moved five places to the right. The result is that there are $5+3=8$ unpaired cations and anions at each electrode,

* Zeit. phys. Chem., 53, 257, 1905; Phil. Trans., A, 206, 101, 1906; Proc. R.S., A, 88, I47, I913; Trans. Chem. Soc., 105, I820, I914. 


\section{CORRECTION OF TRANSFERENCE NUMBERS OF IONS :}

or, in other words, if $\mathrm{U}$ and $\mathrm{V}$ are the respective velocities of the ions, $\mathrm{U}+\mathrm{V}$ is proportional to the number of ions of each kind liberated at each electrode. To apply the above reasoning it must be noted that the passage of one equivalent of electricity $(96,500$ coulombs) as a current means in conventional language the discharge of one equivalent of positive electricity with an equivalent of cations at the cathode and one equivalent of negative electricity with an equivalent of anions at the anode.

Let--

$\mu=$ the concentration of the solute in gram equivalents per c.c. of solution;

$\boldsymbol{a}=$ the ionisation coefficient ;

$\mu a=$ the concentration of ions of either kind;

$\mu(\mathrm{I}-\boldsymbol{a})=$ the concentration of the un-ionised solute ;

$q=$ the charge of electricity of either sign per equivalent of ions $(96,500$ coulombs) ;

$\mathrm{U}=$ velocity of cations relative to the trough in $\mathrm{cm} . / \mathrm{sec}$;

$\mathrm{V}=$ velocity of anions.

Referring now to Fig. 3, let us consider the movement across a mid-

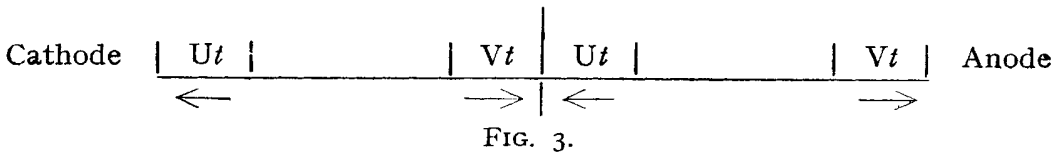

section of the trough. In $t$ seconds, all the cations in a space $U t$ will have crossed the mid-section to the left and all the anions in a space $\mathrm{V} t$ will have crossed to the right, carrying with them their charges. The ionic equivalents in these spaces are $\mu a \mathrm{U} t$ and $\mu \alpha \mathrm{V} t$ respectively, so that the quantity of electricity transported by the ions across the mid-section is

$$
\mu a t(q \mathrm{U}+q \mathrm{~V}) \text { coulombs, }
$$

and therefore, since I coulomb per second is an ampere, we have

$$
k=\mu a(q \mathrm{U}+q \mathrm{~V}) .
$$

The equivalent conductivity $\lambda$ is the specific conductivity $\kappa$ divided by $\mu$, the number of equivalents of solute per c.c. Hence, putting $q \mathrm{U}=u$ and $q \mathrm{~V}=v$, we get the usual, expression

$$
\lambda=\alpha(u+v) .
$$

$u$ and $v$ are the true "mobilities" of the ions, that is, numbers proportional to the actual velocities. The numbers usually tabulated as mobilities are $a u$ and $\alpha v$, whose sum makes up $\lambda$.

Consider now what has taken place in the space $U t$ next the cathode. All the cations originally in space $U t$, amounting to $\mu a \mathrm{U} t$ equivalents, will have moved up to the cathode. But the total current is $\mu a(q \mathrm{U}+q \mathrm{~V})$. Hence, in accordance with Faraday's law, the total liberation of cations in time $t$ will be not merely $\mu \alpha \mathrm{U} t$ equivalents, but $\mu a(\mathrm{U}+\mathrm{V}) t$ equivalents. So that we have liberated at the cathode not merely the equivalent $\mu \alpha U t$ of cations existing originally in the space $U t$, but a further quantity of cations, $\mu a \mathrm{~V} t$. These additional cations come from the ionisation 
of the originally un-ionised portion of the electrolyte present near the cathode, and become ionised near that surface as the cation concentration in that space is diminished by the discharge of cations and the anion concentration in the same space is diminished owing to movement of the anions in that space to the right Similar observations apply to the anode, where a quantity $\mu \boldsymbol{\alpha}(U+V) t$ anions is also liberated, although only $\mu a \mathrm{~V} t$ anions originally existed in the space $\mathrm{V} t$ next the anode.

Referring now to the Hittorf numbers, the numbers usually tabulated are those of the anion, which we may designate by $n_{2}$. The corresponding numbers for the cation may be labelled $n_{1}$, so that-

$$
n_{\mathrm{I}}+n_{2}=\mathrm{I} \text {. }
$$

Hittorf's experimental figures were

(a) the weight of cation discharged at the cathode.

(b) the loss of cation on the cathode side.

The figure he determined was

$$
n_{\mathbf{r}}=\frac{\text { weight of cation transported into cathode compartment }}{\text { weight of cation deposited at cathode }} .
$$

Now, the loss of cation in the cathode compartment=weight of cation deposited--weight of cation transported.

that is to say-

$$
\therefore n_{\mathrm{I}}=\mathrm{I}-\frac{\text { loss of cation in cathode compartment }}{\text { weight of cation deposited at cathode }} \text {, }
$$

$$
n_{2}=\frac{\text { loss of cation in cathode compartment }}{\text { weight of cation deposited }}
$$

Now, these experimental values of $n_{1}$ and $n_{2}$, determined by Hittorf and others, represent the same physical quantities as they did before the question of water transport was thought of-i.e. the ratio experimentally determined of the loss of ions in one compartment to weight or that kind of ion discharged at the electrode. It will be better to keep the letters $n_{1}$ and $n_{2}$ to designate these experimental figures, and to call them still the Hittorf numbers. The difference which the consideration of water transport by the ions makes is one of interpretation. Neglecting all minor volume considerations, the original misinterpretation of the figures in terms of the ionic velocities was

$$
n_{\mathrm{r}}=\frac{\mathrm{U}}{\mathrm{U}+\mathrm{V}} \quad n_{2}=\frac{\mathrm{V}}{\mathrm{U}+\mathrm{V}}
$$

To arrive at the true relation of $n_{I}$ and $n_{2}$ to the ionic velocities we must have regard to the differences in the volumes of the ions, including the water which they carry. We shall still neglect certain minor volume changes, which should be briefly indicated. Taking a solution of $\mathrm{LiCl}$ to illustrate the matter, each ion may be regarded as consisting of the ionic nucleus, together with a number of water molecules travelling with it. The mean density of the associated water is greater than the mean density of water, and on the discharge of the ion (say the $\mathrm{Li}$ ion into a mercury cathode) some volume change necessarily takes place. But this volume change will be small in relation to the concentration changes, and will be neglected. Furthermore, the $\mathrm{Cl}$ ion denuded of 


\section{CORRECTION OF TRANSFERENCE NUMBERS OF IONS :}

its water passes out of the solution. But as the volume of the $\mathrm{Cl}$ in the ion is only a fraction of the total volume of the ion, this again will be neglected in our approximation. Furthermore, during the course of an experiment, as ions are discharged other ions are formed to keep up the proportion of ionised solute, which again causes small volume changes. Also, as the electrolyte becomes more dilute the proportion of ionised solute increases. These changes will also be neglected in our approximation. What we are really seeking is to find to a first approximation what is the effect of the water carried by the ions on the mobilities. I.et

$\mathrm{C}=$ the volume in c.c. of one equivalent of cations,

$\mathrm{A}=$ the volume in c.c. of one equivalent of anions.

The volume of the cations in the space $U t$ which cross the mid-section to the left in time $t$ is therefore

$$
\mu a \mathrm{U} t \mathrm{C}
$$

and of the anions in the other direction?

$$
\mu a \mathrm{VtA} \text {. }
$$

The equation of continuity, or, in other words, the condition that the trough should still be equally filled at all parts, demands a shifting of the neutral electrolyte to make up for the difference in the ionic volumes passing the mid-section. Hence, assuming that VA is greater than UC, there must be a shifting from right to left of a volume $\mu \alpha(\mathrm{VA}-\mathrm{UC}) t$ of neutral electrolyte in time $t$.

This neutral electrolyte contains $\mu(\mathrm{I}-\alpha)$ equivalents of solute per c.c., so that there is a shifting in time $t$ from right to left of

$$
\mu^{2} \alpha(\Sigma-a)(\mathrm{VA}-\mathrm{UC}) t
$$

equivalents of un-ionised solute, which for the purpose only of reckoning the concentration we may consider as anions and cations.

There is therefore on the cathode side, in time $t$

A loss of $\mu a(\mathrm{U}+\mathrm{V}) t$ equivs. of cations discharged at the cathode.

A gain of $\mu a \mathrm{U} t$ equivs. of cations carried over the mid-section.

A gain of $\mu^{2} \alpha(\mathrm{I}-\alpha)(\mathrm{VA}-\mathrm{UC}) t$ equivs. of cations in the neutral electrolyte carried over.

That is to say, there is a total loss in time $t$ of

$$
\mu a \mathrm{~V} t-\mu^{2} a(\mathrm{I}-a)(\mathrm{VA}-\mathrm{UC}) t
$$

equivalents of cations on the cathode side.

Hence, to express Hittorf's experimental number $n_{2}$ in terms of the velocities we have-

$$
\begin{aligned}
n_{2} & =\frac{\text { loss of cation in cathode compartment }}{\text { amount of cation deposited }} \\
& =\frac{\mu a V t-\mu^{2} \alpha(\mathrm{I}-\boldsymbol{a})(\mathrm{VA}-\mathrm{UC}) t}{\mu \boldsymbol{\mathrm { U }}+\mathrm{V}) t} \\
& =\frac{\mathrm{V}-\mu(\mathrm{I}-\boldsymbol{a})(\mathrm{VA}-\mathrm{UC})}{\mathrm{U}+\mathrm{V}}
\end{aligned}
$$


and similarly-

$$
n_{\mathbf{1}}=\frac{\mathrm{U}+\mu(\mathrm{I}-\boldsymbol{a})(\mathrm{VA}-\mathrm{UC})}{\mathrm{U}+\mathrm{V}}
$$

whence, as can easily be seen--

$$
\begin{gathered}
\frac{\mathrm{U}}{\mathrm{V}}=\frac{n_{\mathrm{I}}-\mu(\mathrm{I}-\boldsymbol{a}) \mathrm{A}}{n_{\mathrm{z}}-\mu(\mathrm{I}-\boldsymbol{a}) \mathrm{C}} \\
\overline{\mathrm{U}}=\frac{n_{\mathrm{I}}-\mu(\mathrm{I}-\boldsymbol{a}) \mathrm{A}}{\mathrm{U}+\mathrm{V}}=\frac{\mathrm{I}-\mu(\mathrm{I}-\boldsymbol{a})(\mathrm{C}+\mathrm{A})}{\mathrm{V}} \\
\frac{n_{2}-\mu(\mathrm{I}-\boldsymbol{a}) \mathrm{C}}{\mathrm{U}+\mathrm{V}}=\frac{\mathrm{I}^{2}-\mu(\mathrm{I}-\boldsymbol{a})(\mathrm{C}+\mathrm{A})}{}
\end{gathered}
$$

-which are the true values of the mobilities.

For approximate calculation down to normal dilution we may expand and neglect squares of $\mu(\mathrm{I}-\boldsymbol{a})$, which gives

$$
\begin{aligned}
& \frac{\mathrm{U}}{\mathrm{U}+\mathrm{V}}=n_{\mathrm{I}}+\mu(\mathrm{I}-a)\left(n_{\mathrm{I}} \mathrm{C}-n_{2} \mathrm{~A}\right) \\
& \frac{\mathrm{V}}{\mathrm{U}+\mathrm{V}}=n_{2}-\mu(\mathrm{I}-a)\left(n_{\mathrm{I}} \mathrm{C}-n_{2} \mathrm{~A}\right) .
\end{aligned}
$$

These, then, are the expressions which are proportional to the true mobilities of the ions, instead of the actual Hittorf numbers $n_{1}$ and $n_{2}$.

It will be found that the correction thus introduced is less than the order of the experimental errors down to normal concentration. 'To

\begin{tabular}{|c|c|c|c|c|c|c|}
\hline$m$. & $n_{2}$ & $\mathrm{I}-\alpha$ & c. & A. & $n_{\mathrm{I}} \mathrm{C}-n_{2} \mathrm{~A}$ & $\begin{array}{c}\mu(\mathrm{I}-a) \\
\times\left(n_{1} \mathrm{C}-n_{2} \mathrm{~A}\right) .\end{array}$ \\
\hline$\cdot \mathrm{OI}$ & .63 & $\cdot 075$ & 303 & 86 & $5^{8}$ & $\cdot \mathrm{OO}_{4}$ \\
\hline$\cdot 05$ & $\cdot 67$ & $\cdot 1_{47}$ & 287 & 82 & 40 & .005 \\
\hline$\cdot{ }_{I}$ & $\cdot 69$ & $\cdot 192$ & 269 & 77 & 30 & .006 \\
\hline$\cdot 2$ & $\cdot 7 \mathrm{I}$ & $\cdot 246$ & 244 & $7^{2}$ & 20 & $\cdot 005$ \\
\hline$\cdot 5$ & $\cdot 73$ & $\cdot 328$ & I9I & 62 & 7 & $\cdot 00_{4}$ \\
\hline I & $\cdot 739$ & $\cdot 397$ & 152 & 53 & I & .003 \\
\hline
\end{tabular}
test this we may take the extreme case of I.iCl, where there is a great disparity between the ionic volumes.

In the following table are set out for various values of $m=\mathrm{I}$,000 $\mu$, the values of $n_{2}$ as given by Kohlrausch, the approximate values in c.c.

Table of Corrections of $\mathrm{LiCl}$ Mobilities.

of $\mathrm{C}$ and $\mathrm{A}$, which are the volumes of one equivalent of each kind of ions at different dilutions calculated by the method given in the former papers, the values of $(\mathrm{I}-\boldsymbol{\alpha})$, and in the last column the calculated corrections which must be applied to make the experimental Hittorf number $n_{2}$ proportional to the true ionic mobility. It will be seen that down to normal dilutions the correction is almost inappreciable. Beyond this point the correction changes sign and the factor $\mu(\mathrm{I}-\boldsymbol{\alpha})$ becomes larger, so that the correction will become sensible. For calculation beyond this point the unexpanded expressions should therefore be used. 


\section{O CORRECTION OF TRANSFERENCE NUMBERS OF IONS}

It will be seen that the nentral electrolyte has a constant velocity

$$
\mu a(\mathrm{VA}-\mathrm{UC})
$$

from right to loft across the mid-section. From this it would appear at the first glance that in order to calculate the ionic sizes with the aid of Stokes's law, according to the method of the former papers, it would be necessary to take the velocities relative to the electrolyte as the basis of the calculation. The case is a complex one, since it is not the case of a body moving in a liquid at test, which is that contemplated by Stokes's law, but a body moving relatively to a fluid consisting of three parts moving at different velocities, viz.--

Cations moving to left;

Anions moving to right;

Neutral electrolyte moving to left.

Each of these constituent parts also has a different viscosity. But since the mean velocity of all three parts together is zero, and the mean viscosity of the three constituents is the viscosity of the electrolyte, it appears that we may still take the actual velocities $U$ and $V$ in relation to the trough and the actual viscosity of the whole as the basis for an approximate calculation of the ionic sizes.

St. Swithin's, Hendon, January 6, I9r9. 